Classification

Physics Abstracts

$29.25 \mathrm{~B}-79.70-07.80-41.85-42.40 \mathrm{~K}$

\title{
Point source physics: Application to electron projection microscopy and holography
}

\author{
Roger Morin \\ CRMC2-CNRS, Campus de Luminy, Case 913, 13288 Marseille Cedex 9, France \\ (Received July 4; accepted November 18, 1994)
}

\begin{abstract}
Résumé. - Des émetteurs de champs ultra-fins peuvent produire des faisceaux d'électrons de basse énergie. Cette note présente les résultats expérimentaux récents qui démontrent le fort couplage entre les propriétés atomiques de la source et celles du faisceau d'électrons qui en est émis. Ces expériences comprennent un travail à l'échelle atomique sur la source ainsi qu'une caractérisation des ondes électroniques émises au travers d'expériences d'holographie et d'interférométrie.
\end{abstract}

\begin{abstract}
Ultra-sharp field emitters are able to produce low energy electron beams. This paper presents recent experiments demonstrating the strong coupling between the atomic properties of the source and the properties of the associated electron beam. These experiments include atomic scale work on the source as well as a characterization of tle emitted electron wave through projection holography and interferometry experiments.
\end{abstract}

\section{Introduction.}

It may appear somewhat surprising to say that the wave properties of low energy quasi-free electrons (kinetic energy $<$ a few tens $\mathrm{eV}$ ) are poorly known. However, to our knowledge, the only experiments which directly demonstrate such properties are diffraction experiments on crystal surfaces at an energy above about $50 \mathrm{eV}$. One reason for the absence of experiment in this field is related to the lack of correct electrooptical system in this energy range which prevents from the magnification of interference patterns (the wavelength of electrons at these energies are 1 to a few Angstroms) that might be expected from classical optics experiments.

One way to go around this problem came some years ago with the fabrication of very bright point sources emitting low energy electrons [1]. These sources are ultra-sharp field emission tips and their very end can be visualized by field ion microscopy. At that time, an obvious interest comes from the new-born scanning tunnelling microscopy community but it is the incorporation of these sources in a projection microscope set-up [2], which indeed opens a door towards basic experiments involving low energy electrons. As shown in figure 1, this simple arrangement does 


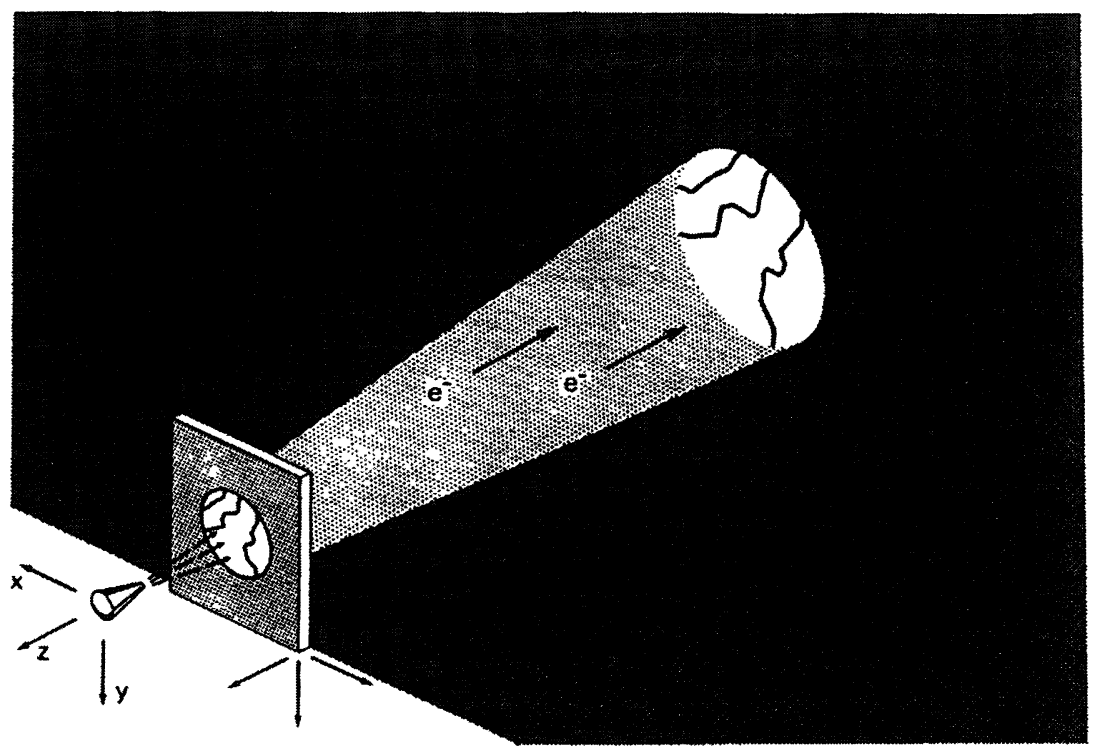

Fig. 1. - Electron point source microscopy.

not use any lens and it is only the ratio of a macroscopic distance (source to screen distance) to a microscopic one (source to object distance) which insures the necessary magnification. Obviously, this set-up is also a microscope which provides shadows of the object at low magnification and holograms of the object at higher magnification (indeed this set-up reproduces the initial idea of Gabor [3] with a physical point source in place of the focus of a convergent high energy electron beam).

The important point which has to be realized is that getting rid of the lens is intimately related to the concept of "point source" i.e. working with electrons prepared in a well defined state. This state would ideally be a divergent monochromatic spin polarized wave originating from a well defined point in space. At the present time, all these quantum numbers are not controlled, for instance spin variable is ignored. But a set of recent experimental results which aim to improve or precise the characteristics of this statistical ensemble of states have been obtained. These results concern the influence of the arrangement and of the nature of the atoms of the source, and the characterization of the electron wave through some interference and interferometry measurements.

\section{The atomic structure of the source and the classical properties of the electron beam.}

As it was previously mentioned the source we are interested in is an ultra-sharp tip emitting electrons by field emission i.e. when a field of a few $\mathrm{V} / \mathrm{nm}$ is applied to the tip surface (the tip is the cathode). One significant difference with other studies involving an electron source is that the atoms of the surface of the tip apex can be "seen" by field ion microscopy (by field ionizing noble gas atoms in the close vicinity of the tip). The early experiments [1] showed that the apex of the tip is made of a few atoms and it is quite natural to think that the nature and the arrangement of these atoms should play an important role on the electron beam properties. However, it is the experiments described hereafter which directly demonstrate such effects. 
2.1 The NATURE OF THE ATOMS OF THE SOURCE AND THE ENERGY SPREAD OF THE ELECTRON STATES. - In order to change the nature of the atoms of the source, different approaches can be considered, for instance changing the material the tip is made of. However preparation techniques are very difficult and it was thought that it would be much easier to deposit foreign atoms on the surface of a tip that was "conventionally" prepared i.e. etched W (111) oriented wire. The deposited atoms are chosen to be alkalis because of their high electropositivity which, from a long time, are known to strongly decrease the work function of $\mathrm{W}$. This deposition is done in vacuum after preparing different atomic arrangements at the tip apex which are characterised by their field ion patterns. The same imaging techniques cannot be applied to visualize the alkali atoms which are field desorbed at lower voltages than the voltage necessary to ionize the imaging gas. However, it was shown [4] that the adsorption process of one single alkali atom is clearly detectable through the increase of the electron field emission current. While the mean increase of the current on a one atom adsorption event is about $40 \%$, up to $600 \%$ increase for the same process was observed. This huge effect compared to similar studies on standard (bigger) field emission tip is likely related to the small size of the emission area. The dispersion of the current increases are related to that of the adsorption sites on the tip apex which can be more or less exposed to the field.

The must important consequences of alkali adsorption on the beam appear on the energy properties [4] (Fig. 2). If an ultra-sharp tip is covered with a layer of Cs, the voltage on the tip for a constant field emitted current is decreased a factor of 4 which means that the mean kinetic energy of the electrons can be decreased down to a few tens $\mathrm{eV}$ with an anode placed at a macroscopic distance of the tip (1 to $10 \mathrm{~mm}$ ). A correspondent decrease, from $250 \mathrm{meV}$ down to less than $100 \mathrm{meV}$, of the energy spread of these electrons is observed, using an electron energy analyser.

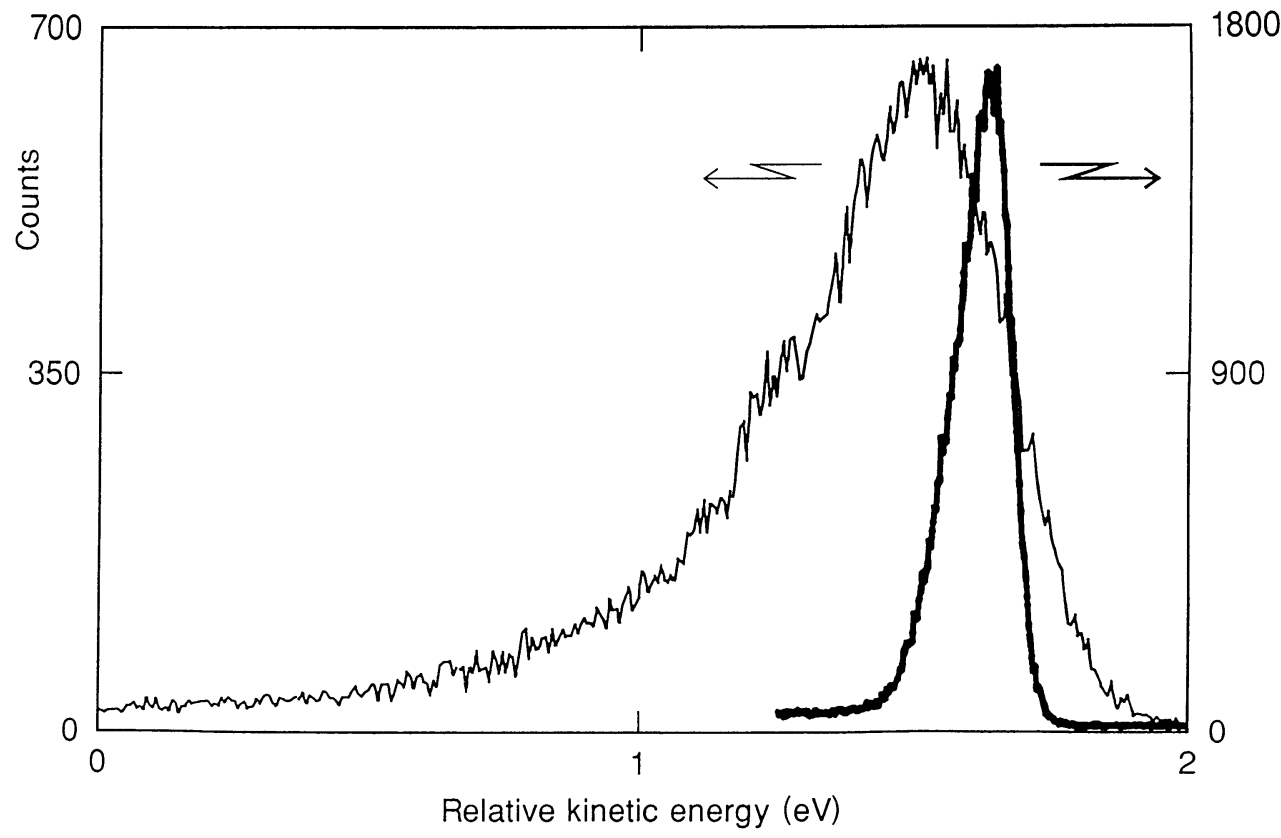

Fig. 2. - Energy distributions of emitted electrons from an ultra-sharp tip: for pure $W$ (thin line, the distribution is centred around $180 \mathrm{eV}$ ) and after Cs adsorption (bold line, the distribution is centred around $48 \mathrm{eV}$ ). 
These two effects can essentially be explained by the well known decrease of the work function from about $4.5 \mathrm{eV}$ down to about $1.65 \mathrm{eV}$ with $\mathrm{Cs}$ adsorption. Similar effects have been observed with other alkali ( $\mathrm{Na}$ and $\mathrm{K}$ ). In conclusion, changing the nature of the atoms of the source can lead to highly monochromatic and very low energy point source beams.

2.2 The ARRANGement of The ATOMS of THE SOURCE AND THE ANGUlar Divergence OF THE BEAM. - One strange behaviour was reported in the first observation of beams emitted from point sources [1]. This is the possibility to observe very low divergence beams, a few degrees angular opening. This looks quite different from what is observed on conventional field emission tips where ten times larger opening angles are usually observed. It was thought for some time that this focusing effect might be the result of the tunnelling of electrons with a wavelength comparable to the size of the tunnelling region [5]. We emphasize that this effect is opposite to the classical description of the transmission of a wave through a hole where the decrease of the hole size increases the divergence of the transmitted wave.

Careful experiments were undertaken to understand this effect [6]. They consist in preparing ultra-sharp tips that can be characterised at the atomic level by field ion microscopy, looking to the opening of the electron beam and back to field ion microscopy, checking that the atomic structure of the tip is unchanged. This study leads to associate the focusing effect to pyramidal shaped ultra-sharp tips only. It was shown that increasing, from 1 to 10 , the number of atoms terminating the tip leaves the opening angle unchanged (Fig. 3). This desagrees with the effect previously described [5] and is understood as the result of patch fields on a nanometer scale. These fields are electrical fields located outside the tip and connect crystal faces of different work function. In the present experimental conditions the associated classical force on an electron is oriented towards the axis of the tip and thus corresponds to a focussing effect.

This study illustrates an atomic scale approach to control the angular dependence of electron

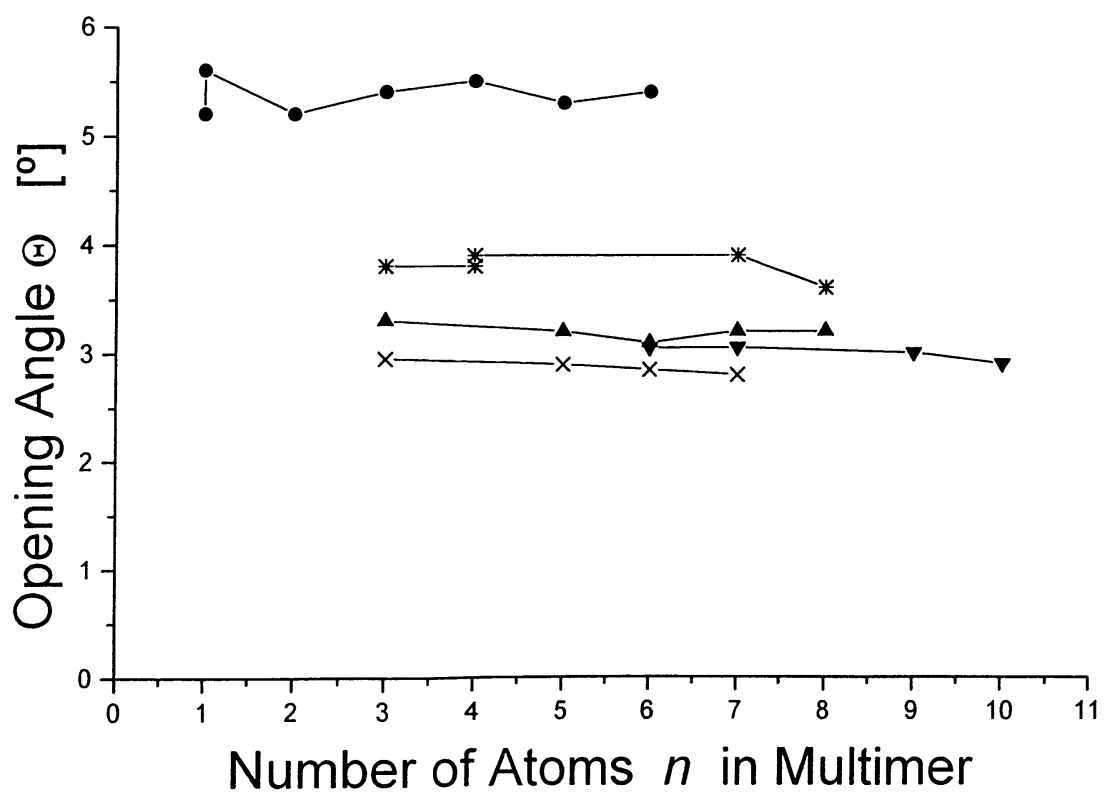

Fig. 3. - Opening angle of the electron beam emitted by pyramidal ultra-sharp tips vs. the number $n$ of adatoms terminating the tip. The four different curves correspond to four different applied fields. 
waves. It is quite different from that one usually used in similar experiments where one select waves using macroscopic devices like diaphragms and lenses from a huge set of different states.

\section{Quantum properties of electron beams emitted from ultra-sharp tips.}

In the previous paragraph, we address properties of the electron beam which do not rely on the wave aspect of electrons. However, there are numbers of questions which require such a description. These questions obviously appear in imaging small objects like in projection microscopy. However we adopt here the reverse point of view which is to consider the projection point source microscope as a tool to characterize the electrons originating from the tip. In this way interference experiments are related to the physics of the source.

Up to now, two different kinds of experiments have been carried out. The first kind to be realized and the simplest one consist in obtaining a Gabor in-line hologram [7-10] which is an interference pattern between the transmitted wave and the wave which is scattered by an object (Fig. 4). Different kinds of objects have been used. All of them are deposited on thin carbon or gold membranes. Although these experiments are very instructive and clearly prove some degree of coherence of the electron beam, the second kind of experiments, although much more difficult to perform experimentally, provides a straightforward information on the electron states.

These experiments $[11,12]$ consist in obtaining interferograms by adding an electrostatic biprism (i.e. a thin electrically charged wire) to the projector set-up (Fig. 5). The effect of the electrostatic field around the wire is to transform the incident divergent wave into two divergent waves (originating from two virtual sources) which interfere on the screen if the distance between the two sources is larger than the wire diameter. Because of the small electron wavelength, in order to get visible fringes, it is however required that these two virtual sources are close enough, less than $1 \mu \mathrm{m}$ apart. These contradictory conditions lead to the use of very thin wires, a few tenths of a micrometer in diameter. The observation of fringes (Fig. 6) is obviously related to the size of

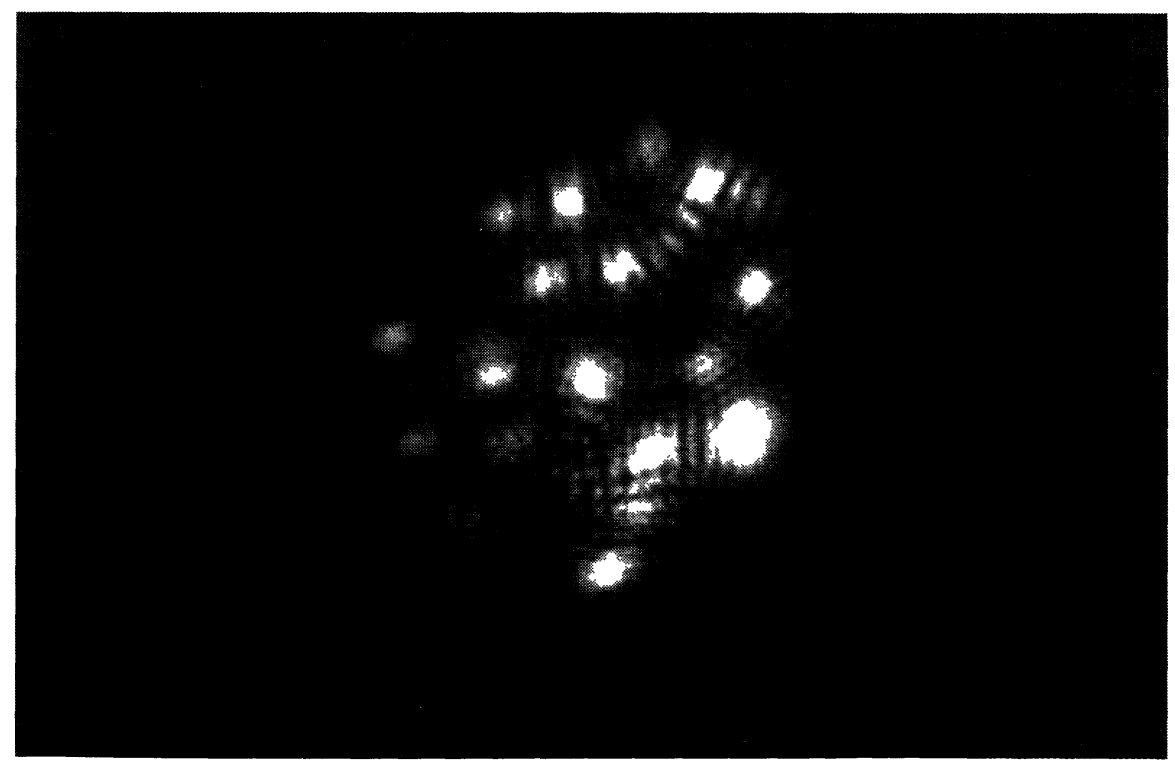

Fig. 4. - Gabor type in line low energy $(94 \mathrm{eV})$ electron hologram of a thin carbon membrane. 


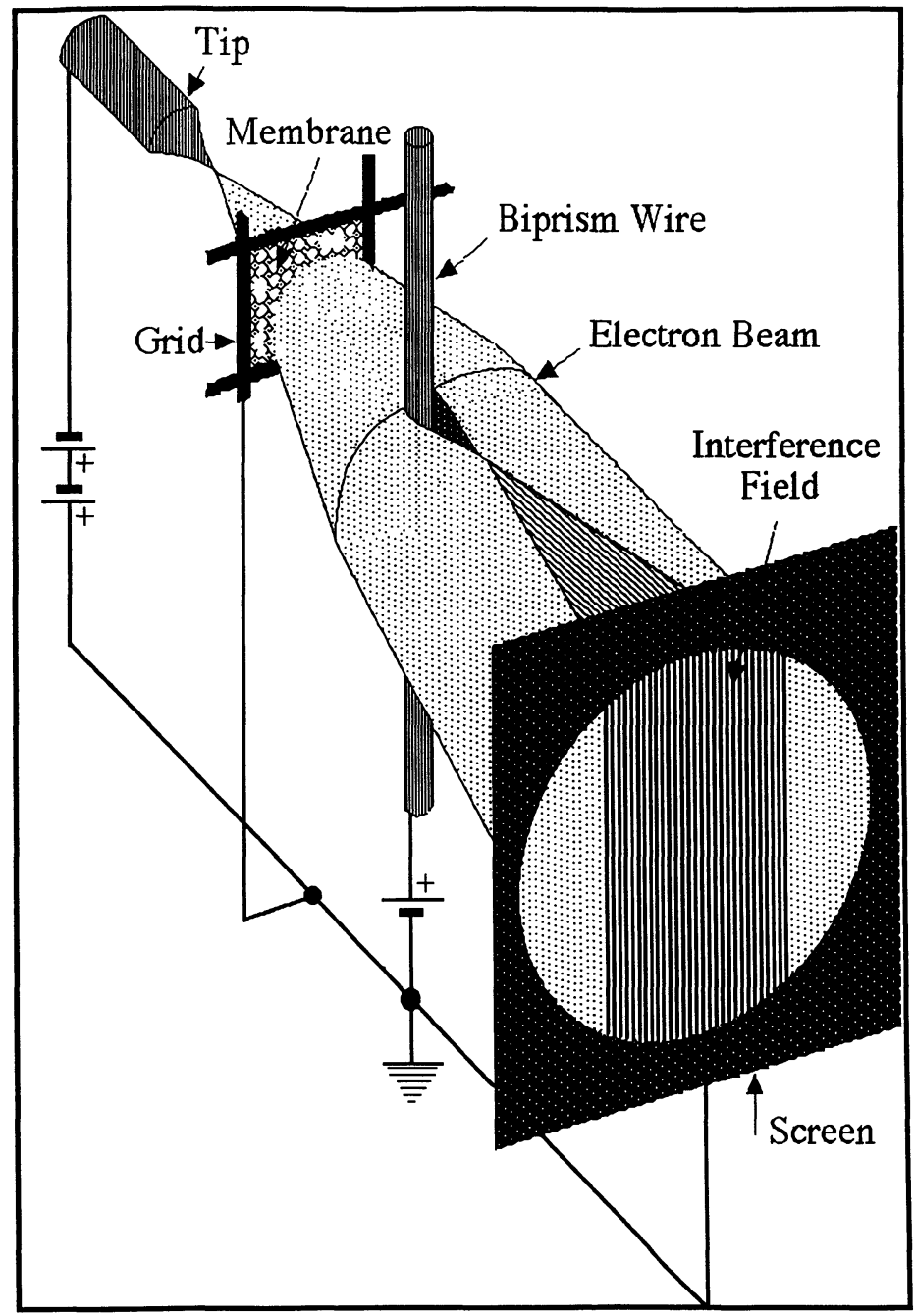

Fig. 5. - Principle of the interferometry experimental arrangement.

the source in the following way. Each electron state produces its own interference pattern on the screen (of course, as this is always the case, the detection of one electron at a given point destroys the interference and it is the similarity of the different wave functions for the successive electrons which allows to see the interferences). Therefore, if this electron state is described by a divergent wave originating from a given point in space, the properties of the ensemble of electron states is described by the distribution of the points of the source i.e. the size of the source. Summing the intensity contributions of each point of an extented source allowed to determine an upper value of the source size from the interference pattern [13]. A radius of $0.16 \mathrm{~nm}$ was found which is clearly of the order of the size of an atom and leads to a brightness of about $10^{9} \AA \mathrm{cm}^{-2} \mathrm{sr}^{-1}$ for $\mathrm{nA}$ current and a few degrees divergence angles [6]. It is worth to emphasize that the determination of this upper limit of the source size is weakly dependent on the intensity measurement in the limit of weak contrast (which is the present case), where only the discernability of fringes is important. 


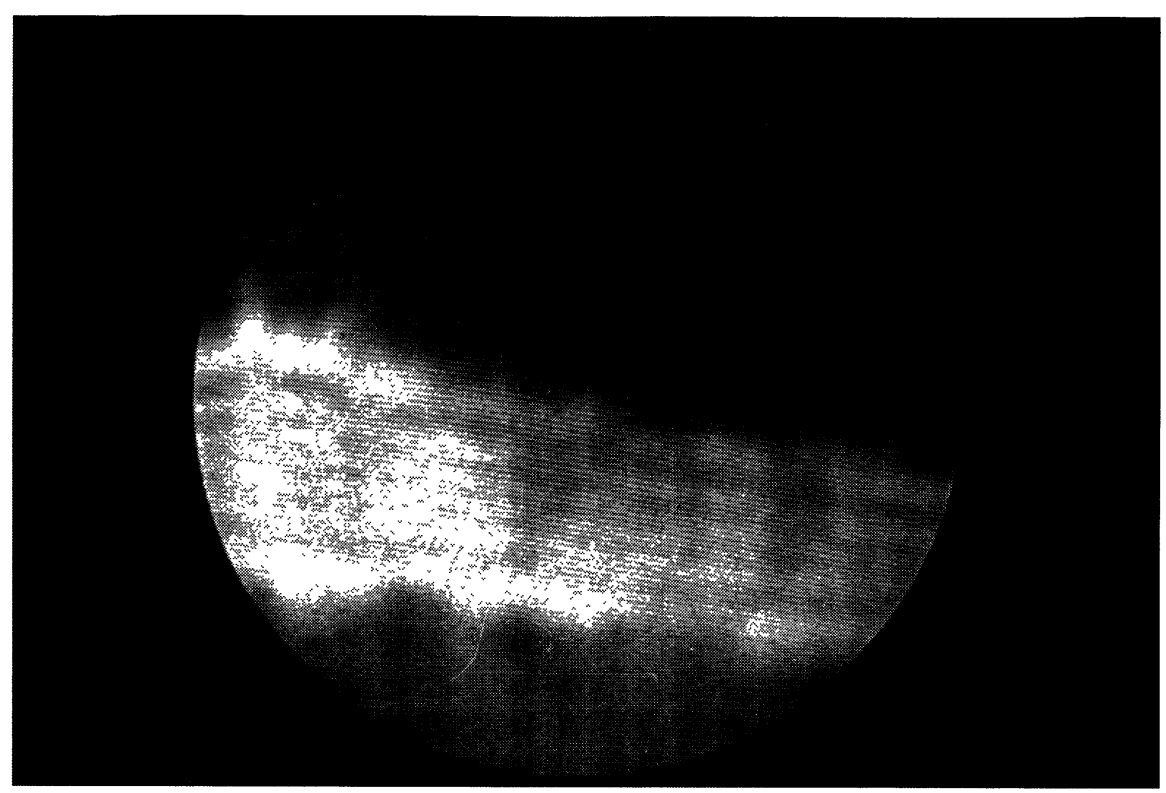

Fig. 6. - Low energy $(57 \mathrm{eV})$ interference pattern obtained with an electrostatic biprism (the fringe spacing is about $0.3 \mathrm{~mm}$ ).

Moreover, the obtained atomic size is quite surprising if we think that, even if a field ion micro graph can show a single atom tip, in the case of electrons emission such a tip is embedded in a tunnel region 1 to $2 \mathrm{~nm}$ thick. This result is a direct answer to the most elementary question since the building of the first electron point source.

\section{Conclusion.}

In the present paper, we have described different experimental approaches which demonstrate that ultra-sharp field emission tips are able to produce low energy monochromatic coherent electron beams originating from a volume in space as small as the size of an atom. These approaches are quite different from those usually used in similar problems where macroscopic devices are used to select a narrow distribution of states from the huge number of states produced from a macroscopic source. In the present case, the atomic scale control of the source and the positioning of submicrometric objects to characterize the emitted electron waves have essentially been used.

\section{References}

[1] Fink H.-W., IBM J. Res. Dev. 30 (1986).

[2] Stocker W., Fink H.-W. and Morin R., Ultramicroscopy 31 (1989).

[3] Gabor D., Nature (London) 161 (1948) 777.

[4] Morin R. and FinkH.-W., Appl. Phys. Lett. 65 (1994) 2362. 
[5] Lang N.D., Yacobi A. and Imry Y., Phys. Rev. Lett. 63 (1989) 1499;

Teckman E., Ciraci S. and Baratoff A., Phys. Rev. B42 (1990) 9221.

[6] Horch S. and Morin R., J. App. Phys. 74 (1993).

[7] Fink H.-W., Stocker W. and Schmid H., Phys. Rev. Lett. 65 (1990).

[8] Fink H.-W., Schmid H., Kreuzer H.J. and Wierzbicki A., Phys. Rev. Lett. 67 (1991).

[9] Morin R., Gargani A., Phys. Rev. B48 (1993).

[10] Spence J.C.H., Quian W. and Melmed A.J., Ultramicroscopy 52 (1993).

[11] Degiovanni A. and Morin R., Proceedings ICEM 13 (Les Editions de Physiques, 1994).

[12] Morin R. and Degiovanni A., J. Vac. Sc. Tech. (1994), in press.

[13] Degiovanni A. and Morin R., submitted to Phys. Rev. Lett. 